\title{
PATHOPHYSIOLOGY
}

UDC 615.03

\section{Application of cellular and artificial membranes in nanomedicine}

\author{
M. A. Galkin \\ First Pavlov State Medical University of St. Peterburg, \\ 6-8, ul. L'va Tolstogo, St. Petersburg, 197022, Russian Federation
}

For citation: Galkin M. Application of cellular and artificial membranes in nanomedicine. Vestnik of Saint Petersburg University. Medicine, 2020, vol. 15, issue 4, pp. 290-299.

https://doi.org/10.21638/spbu11.2020.407

\begin{abstract}
The use of nanoparticles in treatment and diagnostics of a number of disorders is becoming more and more popular. Further investigations are needed for improving the specificity of nanoparticle action, precisely targeted drug delivery, decreasing opsonization of nanoparticles by macrophages. Numerous ways of nanoparticle surface modification have been successfully tested for increasing their therapeutic potential and reducing possible side effects. Nanoparticle encapsulation using plasma membranes of red blood cells as well as other cell types has been recently introduced. This field of translational medicine substantially expands opportunities for nanoparticle application in clinical diagnostics and therapy of cancer, cardiovascular diseases, in vaccine development etc. This review focuses on ways, advantages and disadvantages of using cellular membranes in nanomedicine. Application of artificial lipid membranes in nanoparticles encapsulation is proposed.
\end{abstract}

Keywords: nanoparticles, cell membranes, liposomes.

\section{Introduction}

Drugs administered into human body are nonspecifically distributed in different tissues, which requires injecting big doses of drugs to reach a therapeutic effect resulting in undesirable side effects. Thus a targeted drug delivery is vital. One of efficient ways for drug's target delivery is using nanoparticles, the diameter of which is usually below $100 \mathrm{~nm}[1 ; 2]$. The properties of nanoparticles are dictated by material they are made from as well as their size, form, surface properties, dispersion degree. Extremely small size of nanoparticles or rather very high surface area to volume ratio is especially beneficial in their use. Nanoparticles for biomedical purposes are currently made from a variety of

(C) St. Petersburg State University, 2020 
polymeric materials and minerals, including gelatin, PLGA (poly(lactic-co-glycolic acid)), polyacrylamide, lipids, silicon, gold, ferric oxide etc. [3]. Drug-loaded nanoparticles can passively accumulate in target tissue, such as tumor, due to the enhanced permeability and retention effect [4]. Target delivery can be more precise if nanoparticles have special physicochemical properties including their size, surface charge, hydrophilicity and geometry. On the other hand, synthetic nanoparticles can be toxic [2]. What's more, "naked" synthetic nanoparticles can activate the complement system, they are prone to opsonization and uptake by macrophages. Administration of nanocarriers also increases the risk of anaphylatoxin generation [5]. In order to avoid these side effects, nanoparticles should be somehow chemically modified (functionalized) depending on their specific application and/or target. The surface of nanoparticles can be modified with ligands, polymers, fluorescent labels, enzymes, monoclonal antibodies, aptamers, and other biomolecules. Nanoparticle encapsulation into natural cell membranes has recently become one of the most popular and universal ways of their functionalization.

This review focuses on advantages of using cellular membranes in nanomedicine in treatment and diagnostics of various diseases. Nanoparticles coated by cell membranes can be applied for antimicrobial defense, for creation of more efficient vaccines and so on [6].

A method for nanoparticle modification with artificial proteoliposome membranes is proposed and its advantages/disadvantages are discussed.

\section{Nanoparticle encapsulation into cellular membranes}

High density lipoproteins (HDL) can be regarded as natural nanoparticles. They are composed of a hydrophobic core that contains triglycerides and cholesterol esters and an outer monolayer of phospholipids with some apolipoproteins. These lipoproteins play an essential role in cholesterol transport from peripheral tissues to the liver and its removal from macrophages in atherosclerotic plaques [7]. Cormode et al made an artificial model of HDL, coating nanoparticles with lipids ("HDL"). An APO-I peptide was used instead of APO-I apolipoprotein [8]. The core of "HDL" was filled with inorganic nanocrystals made of gold and iron oxide serving as contrast agents in molecular visualization. The phospholipid crown of the particles contained lipids carrying fluorescent and paramagnetic labels that also served for visualization. These particles can be a valuable tool in multimodal diagnostics that combines detection of emission, computer tomography as well as confocal and transmission electron microscopy. The artificial "HDL" were stable for at least three months, not prone to aggregation and precipitation, exhibited a long lifespan in the circulation. They indeed bound to macrophages accumulating in atherosclerotic plaques, which is promising for their use in molecular diagnostics.

The "HDL" can be used in studies of HDL-cell interactions, their kinetics and thermodynamics. Modulations of these interactions could be achieved by tuning the size of the particles. "HDL" are a promising tool for diagnostics of atherosclerosis as well as other inflammatory conditions: liver pathologies, rheumatoid arthritis, tumors [8].

Liposomes are tiny phospholipid membrane vesicles that represent one of the simplest and most available types of nanoparticles. They can be used for the delivery of drugs both hydrophilic (inside liposomes) and hydrophobic (embedded in the lipid bilayer). The main disadvantage of liposomes is their low stability. Small (diameter less than $100 \mathrm{~nm}$ ) liposomes are prone to fusion because of high surface curvature and high surface tension. 
Coating of liposomes with polyethylene glycol stabilizes them, preventing liposome fusion and plasma proteins binding, resulting in longer lifetime of the preparation in the circulation [9].

A recently proposed encapsulation of nanoparticles into cellular membranes has proved a very promising approach. One of the most available sources of cell membrane is erythrocytes that are abundant in the blood and easy to operate with. Erythrocyte ghosts can be obtained by osmotic shock followed by centrifugation and wash of the precipitate in hypotonic buffer. Combination of ultrasound treatment and extrusion through polycarbonate filters enables to make membrane vesicles of wanted size. The vesicles are then mixed with $70 \mathrm{~nm}$ nanoparticles and the preparation is extruded through polycarbonate filters once again. As a result, nanoparticles encapsulated into erythrocyte membrane are formed. Coating nanoparticles with membranes from other cell types is more complicated, but is based on the same principles as in the case of erythrocytes [10].

Encapsulation into cellular membranes imparts nanoparticles a variety of properties that increase opportunities for their use in diagnostics and treatment of various disorders. Nanoparticles coated with cell membranes are less prone to aggregation, their colloid stability is increased [11]. Some advantages of nanoparticle coating technology and its possible applications in medicine are summarized below.

- Prolongation of nanoparticles' circulation lifetime.

A number of plasma membrane components serve as markers of "self" cells (autoantigens). CD47 in erythrocyte membranes prevents the uptake of erythrocytes by macrophages (a "don't eat me" signal) [12;13]. Other erythrocyte membrane proteins such as C8bp (C8 binding protein) [14], decay accelerating factor (DAF), membrane cofactor protein (MCP), complement receptor 1 (CR1), CD59 and homologous restriction protein (HRP) [15] prevent activation of the complement system [16]. In addition to the proteins, phagocytosis of red blood cells (RBCs) is prevented by negatively charged sialic acids in the glycocalyx [17]. With help of anti-CD47 antibodies the orientation of the RBC membranes on gold nanoparticles was shown to be the same as in the cells. This is related to an asymmetric distribution of the charge of erythrocyte membrane: the outer leaflet of the membrane is charged more negatively than the inner one. Hence the negative surface charge of gold nanoparticles promotes the correct orientation of the coating erythrocyte membrane.

- Use of nanoparticles coated with cell membranes for vaccination.

Antibacterial vaccines are routinely made based on inactivated bacterial cells, bacterial proteins or polysaccharides and inactivated bacterial toxins (toxoids). Widespread use of these agents led to a substantial reduction of mortality worldwide. With that, effective vaccines against Staphylococcus aureus, Helicobacter pylori, Shigella, and Escherichia coli have not been created so far. In addition, the application of antibiotics to fight bacterial infections results in bacterial antibiotic resistance. A promising alternative for making vaccines is encapsulation of nanoparticles in bacterial plasma membranes.

To this end, two alternatives can be applied: 1) vaccination against virulence factors that sequesters bacterial toxins; 2 ) vaccination against pathogens presenting bacterial antigens [18]. Intact toxins embedded in bacterial membranes ("nanotoxoids") cannot exert their pathogenic effect, but can serve for generation of an immune response. Inactivated 
(denatured chemically or thermally) toxins are typically used for vaccine production. Denaturation destroys the three-dimensional structure of a toxin, which changes its antigenic determinants and makes it less immunogenic.

The toxic effect of alpha-hemolysin from Staphylococcus aureus is related to its incorporation into RBC membranes and subsequent cell lysis [19]. A vaccine against alphahemolysin (nanotoxoid) was created based on PLGA (poly(lactic-co-glycolic) acid) nanoparticles coated with RBC membrane. Hemolysin reconstituted into erythrocyte membranes on the surface of nanoparticles retained its antigenic properties and was nontoxic. The mice vaccinated intravenously with the nanotoxoid demonstrated a stronger immune response than the control group vaccinated with denatured hemolysin. Cell membranecoated nanoparticles may serve as a decoy for bacterial toxins that interact with components of the cell membrane.

The other approach is to use bacterial plasma membrane - coated nanoparticles as vaccines. On one hand these formulations have a complete array of antigenic determinants for the immune response. On the other hand the size and form of nanoparticles can be easily controlled, which is essential for an efficient presentation of an antigen to immune cells. As a result, the effect of combination of nanoparticles and bacterial membranes is synergic leading to amplification of antibacterial immune response. The outer membrane vesicles secreted by Gram- negative bacteria, such as E.coli, were used as the source of bacterial membrane [18].

An antibacterial effect was also manifested in case of nanoparticles coated with platelet membranes (PNP). Nanoparticles loaded with the antibiotic vancomycin were more efficient against staphylococcus than the antibiotic in solution. This can be explained by capability of platelet cell membrane components for specific interactions with staphylococci and streptococci [5].

- Application of encapsulated nanoparticles for detoxification.

Unfortunately, critical care units pretty often admit patients that got a dangerous dose of a highly active poison or drug either incidentally or as a result of suicide attempt. Among the most lethal poisons are organophosphorus compounds including insecticides, pesticides and neuroparalytic warfare agents such as sarin and saman. Their toxicity is caused by irreversible inhibition of acetylcholinesterase at negligibly small concentrations $-10^{-7} \mathrm{M}$ or less [20]. Organophosphates phosphorylate the catalytic serine hydroxyl at the active site of acetylcholinesterase leading to accumulation of acetylcholine in synapses and disruption of cholinergic synaptic transmissions. This can result in neurotoxic effects and even death. Development of an effective antidote for this type of compound and other toxic agents continues to be relevant. Pang et al. [21] used nanoparticles coated with RBC membrane for detoxification of the organophosphorus compound dichlorvos. Acetylcholinesterase in erythrocyte membranes on the surface of nanoparticles was able to efficiently bind dichlorvos intravenously injected in mice, protecting the synaptic acetylcholinesterase thereby preventing or mitigating the poisoning. When dichlorvos was injected alone, all the mice used perished within an hour. But upon the combined injection of dichlorvos and nanoparticles coated with $\mathrm{RBC}$ membranes all the mice treated remained alive for at least 16 more days. Thus nanoparticles encapsulated in cellular membranes can be used as effective antidots counteracting diverse toxins and poisons. 
- Application of nanoparticles coated with cell membranes in treatment of autoimmune disorders.

Idiopathic thrombocytopenic purpura is a condition in which autoantibodies attack host platelets, resulting in uncontrolled bleeding that can be fatal. In vitro and in vivo tests on blood coagulation in mice demonstrated that nanoparticles coated with platelet membranes efficiently bind platelet autoantibodies, preventing platelet destruction in the reticuloendothelial system in thrombocytopenia [22].

\section{- Targeted delivery of drugs.}

Nanoparticles coated with cell membranes are able to accumulate in target organ using homotypic and heterotypic adhesive properties of cell membranes. For example, tumor cells express CD44 on their surface [23]. Nanoparticles encapsulated into platelet membranes containing P-selectin mainly accumulate in a tumor (heterotypic adhesion). On the other hand, diverse tumor cells express surface antigens with homophilic adhesive domains such as carcinoembryonic antigen and galectin-3 necessary for formation of multicellular aggregates in tumors. The heterotypic adhesion to the microvascular endothelium and subsequent homotypic aggregation are critical in the generation of metastases in the blood [1].

Cell's capability of heterotypic and homotypic adhesion underlies the use of nanoparticles for precise targeted drug delivery, which, in particular, can be applied in photodynamic therapy for treating cancer.

As an example, PLGA nanoparticles (negative surface charge) were coated with platelet membranes (NP-P) or RBC membranes (NP-R), the nanoparticles being preliminary loaded with the photosensitizer verteporfin [24]. Upon intravenous injection in BALB/c mice with tumor $4 \mathrm{~T} 1$ the nanoparticles were distributed to different organs. Remarkably, the accumulation of the NP-P in the tumors substantially exceeded that of the NP-R. The subsequent laser irradiation (at $680-730 \mathrm{~nm}$, near the absorption maximum of verteporfin in nanoparticles) led to a much more pronounced tumor shrinking in case of the NP-P compared compared with the NP-R. The survival rate of the mice for 35 days starting from the injection was $100 \%$ in case of NP-P, $75 \%$ in case of NP-R, $0 \%$ in case of phosphate buffered saline without NP.

Nanoparticles encapsulated in cancer cell membranes may play a double role. On one hand, they can be used for vaccination against cancer as due to the membrane they carry tumor antigens, and can also be supplied by immunologic adjuvant. As a result, upon interaction with antigen-presenting cells these nanoparticles are able to cause an antitumor immune response. On the other hand, carrying the same adhesion molecules as the membranes of tumor cells the nanoparticles are capable of specific binding to these cells owing to homotypic interaction [25].

One of anticancer vaccines actively used is Provenge (Sipuleucel-T). It can be applied for treatment of adenoma. For generation of this vaccine prostate acid phosphatase is used [26].

As noted above, nanoparticles coated with tumor cell membranes can improve the efficiency of vaccination against cancer due to various membrane -bound cancer antigens. In addition, the nanoparticles can be loaded with adjuvant.

It is also possible to combine platelet and $\mathrm{RBC}$ membrane when coating nanoparticles [27]. The nanoparticles encapsulated in platelet/RBC membranes exhibited proper- 
ties typical for both platelet and erythrocyte membranes. Thus, their applications in the clinic can be broader than in the case of nanoparticles coated with membranes from single source.

\section{- Issues that affect the application of nanoparticles encapsulatedin cell membranes.}

This review clearly shows that the technology of encaplsuting nanoparticles in cell membranes is very promising for the treatment and diagnostics of a wide range of disorders. With that, there are some drawbacks of this type of nanoparticles:

- laborious procedures of obtaining cell membranes. RBC membranes are relatively easily isolated and available. The isolation of platelet membranes is much more complicated and costly, in particular, it requires protease inhibitors, and platelet aggregation should be avoided. Most other cell types can be obtained from the respective cell lines.

- very low yield of nanoparticles coated with cell membranes.

- cell membranes contain both "useful" proteins that serve for nanoparticle binding to therapeutic targets and a big number of other proteins that may generate immune reactions.

- the absence of clear criteria for estimation of the quality of biologic material used for preparation of cell membrane encapsulated nanoparticles; the safety of these preparations is not completely proven [28].

\section{Possibilities of coating nanoparticles with modular proteoliposome membranes}

Artificial lipid membranes can be used to mimic the natural cell membranes. This approach allows to use any suitable types of lipids for controlled reconstitution of membrane proteins, both recombinant and artificially constructed, into liposomes. For reconstitution of a membrane protein in liposomes it should be first mixed with a detergent and unilamellar liposomes (lipid bilayer) with subsequent removal of the detergent with help of gel-filtration, dialysis etc. [29].

The formation of proteoliposomes usually includes the following steps:

1) extraction of membrane proteins using detergents;

2) purification of the protein of interest;

3) preparation of unilamellar liposomes;

4) solubilization of the liposomes with detergents and formation of lipid-detergent micelles with subsequent adding the protein. As a result, mixed protein/lipid/ detergent micelles;

5) removal of detergents and formation of proteoliposomes.

The method described works well for small liposomes (diameter $=30-200 \mathrm{~nm}$ ), but is not that efficient in case of large $(200-1000 \mathrm{~nm})$ and giant (more than 1 micron) liposomes.

Proteoliposomes containing different proteins may fuse due to oppositely charged lipids [30-33].

Liposomes made mainly of phosphatidylcholine were charged due to $10-40 \%$ either negatively or positively charged lipids (anionic or cationic liposomes, respectively). A pro- 


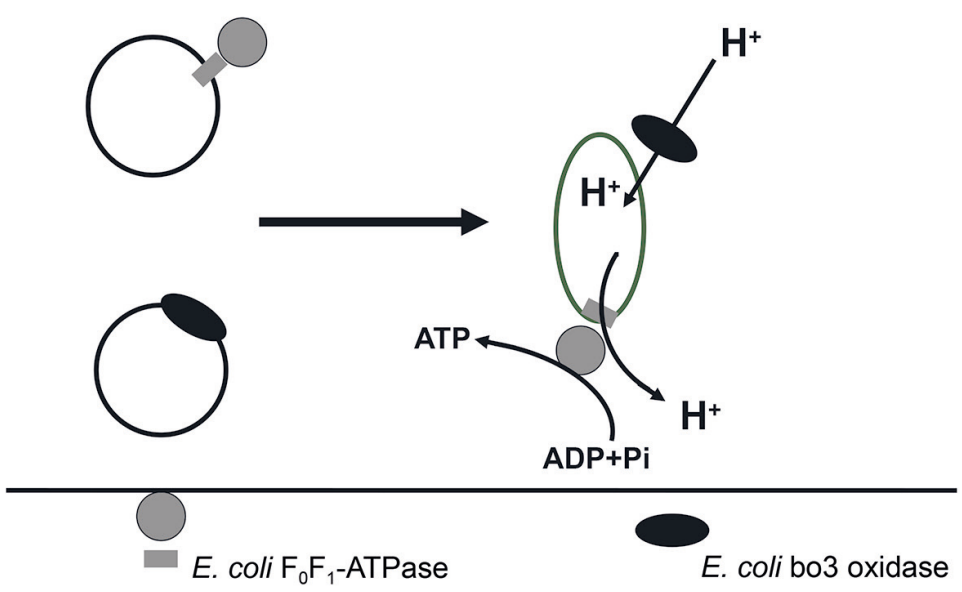

Fig. 1. Fusion of proteoliposomes containing components of the bacterial respiratory chain. E. coli $\mathrm{F}_{0} \mathrm{~F}_{1}$-ATPase and bo3 oxidase were reconstituted into anionic and cationic liposomes, respectively. The efficiency of proteoliposomes' fusion was estimated by adding the substrates of both enzymes to the incubation medium. Proton gradient generated by bo3 oxidase caused formation of ATP detected with the luciferin-luciferase system. See the text for further explanation

ton gradient generator, E. coli complex IV ( $\mathrm{bo}_{3}$ oxidase), was reconstituted in the cationic liposomes, while E. coli $\mathrm{F}_{0} \mathrm{~F}_{1}$-ATPase was reconstituted in the anionic liposomes [30; 31]. The two proteoliposome preparations were mixed. Fusion of the proteoliposomes was completed in 5-10 min, which could be confirmed by detecting ATP synthesis (Fig. 1). The efficiency of proteoliposomes fusion critically depended on the fraction of charged lipids, temperature and liposome size. Smaller liposomes fuse faster due to higher surface curvatures. A drawback of this method is using cationic lipids that do not occur naturally, so it is necessary to test effects of these lipids on the activity of membrane proteins.

The modular (proteo)liposomes resulting from fusion of oppositely charged (proteo) liposomes can be exploited for coating nanoparticles (Fig. 2).

This putative procedure could include the following steps.

- First, preparation of proteoliposomes containing at least two types of proteins: 1) those functioning as a "don't eat me" signal, like CB47 from RBC; 2) those specifically interacting with the target cells. The binary system is possible when the proteins are first reconstituted into oppositely charged liposomes that can fuse, forming proteoliposomes containing both proteins. In case of the ternary system, both types of proteins are initially reconstituted into positively charged liposomes. Then the two preparations of proteoliposomes containing one of the proteins fuse with negatively charged large or giant liposomes.

- Next step: the modular proteoliposomes obtained in step one are mixed with nanoparticles that are coated with the proteoliposomes membranes by means of ultrasound or extrusion through polycarbonate filters.

Some advantages of the proposed approach are as follows.

- Purified (recombinant) proteins can be used. Modern purification procedures allow to get big amounts of homogenous highly active protein. In particular, the 
a) liposomes

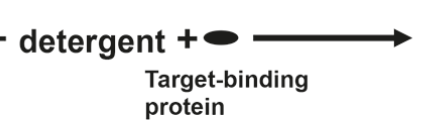

Detergent removal

(dialysis or gel filtration)

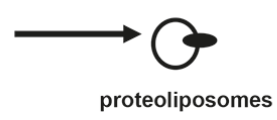

b)
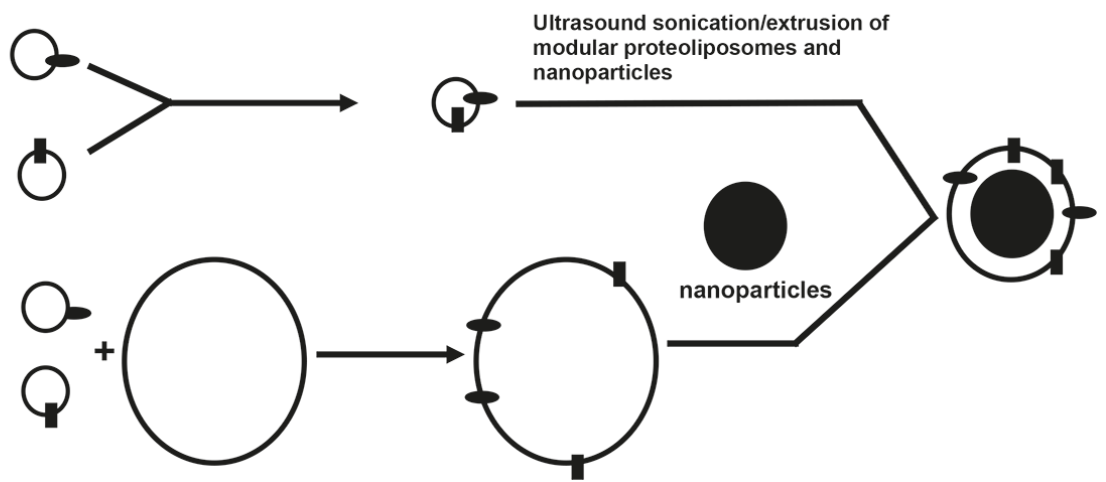

Fig. 2. Diagram of putative encapsulation of nanoparticles into fused proteoliposome membranes. a) obtaining proteoliposomes containing a target tissue binding protein; b) fusion of oppositely charged proteoliposomes containing a target tissue binding protein as well as a "don't eat me signal" protein, with formation of combined proteoliposomes (binary system); c) preparation of positively charged proteoliposomes containing either a target tissue binding protein or a "don't eat me signal" protein followed by fusion of both types of proteoliposomes with negatively charged large or giant liposomes. Encapsulation of nanoparticles can be carried out by treatment of the combined proteoliposomes by ultrasound or extrusion using polycarbonate filters. See the text for further explanation

use of affinity chromatography (for example, Ni-NTA agarose that is capable of tight binding of hexahistidine tags in recombinant proteins) facilitates preparation of a homogenous pure protein. As noted above, the cell membranes contain both "necessary" proteins that serve for binding to the target cells and a lot of other proteins that can induce immune responses. Proteoliposomes, contrary to natural cell membranes, can be enriched in the "necessary" proteins, resulting in a more tight and specific interactions of the nanoparticles with their targets. In addition, other membrane proteins do not interfere with those interactions. What's more, cell material is often not enough for clinically meaningful nanoparticle encapsulation;

- Immense opportunities for selection of optimal lipids (and their combinations) based on chemical properties, charge and other parameters for exertion of the required biomimetic effect.

- Possibility for standardization of the material created and clear criteria for its quality control.

Concerning possible drawbacks of application of artificial membrane vesicles. One of them is necessity for purification of homogenous proteins. Protein purification is often very difficult, mutistep and expensive process. In the case of recombinant proteins a tissue cell culture with strict sterility and safety rules is required. 


\section{Conclusion}

This review describes the ways of nanoparticle encapsulation in the cell membranes as well as possibilities of using this technology in modern medicine, both in therapy and diagnostics. Coating nanoparticles with cell membranes prolongs their circulation lifetime, increases their colloidal stability. Nanoparticles encapsulated in cell membranes can be used as efficient vaccines against infections and tumors. They also promote precise targeted delivery of drugs and can be used in treatment of autoimmune disorders. The encapsulated nanoparticles have potential for detoxification of natural (produced by microbe) and chemical toxins. It is evident that the number of applications of these nanoparticles will expand. Using artificial lipid membranes mimicking cell membranes in coating nanoparticles is proposed.

\section{References}

1. Gao W., Zhang L. Coating nanoparticles with cell membranes for targeted drug delivery. Journal of Drug Targeting, 2015, vol. 23, no. 7-8, pp. 619-626.

2. Murthy S.K. Nanoparticles in modern medicine: state of the art and future challenges. International Journal of Nanomedicine, 2007, vol. 2, no. 2, pp. 129-141.

3. Zhai Y., Su J., Ran W., Zhang P., Yin Q., Zhang Z., Yu H., Li Y. Preparation and application of cellmembrane-camouflaged nanoparticles. Theranostics, 2017, vol. 7, no. 10, pp. 2575-2592.

4. Yuan F., Dellian M., Fukumura D., Leunig M., Berk D. A., Torchilin V.P., Jain R.K. Vascular Permeability in a Human Tumor Xenograft: Molecular Size Dependence and Cutoff Size. Cancer Research, 1995, vol. 55, pp. 3752-3756.

5. Hu C.-M.J., Fang R.H., Wang K.-C., Luk B. T., Thamphiwatana S., Dehaini D., Nguyen P., Angsantikul P., Wen C. H., Ashley V., Kroll A. V., Carpenter C., Ramesh M., Qu V., Patel S., Zhu J., Shi W., Hofman F. M., Chen T.C., Gao W., Zhang K., Chien S., Zhang L. Nanoparticle biointerfacing via platelet membrane cloaking. Nature, 2015, vol. 526, no. 7571, pp. 118-121.

6. Kroll A., Fang R.H., Zhang L. Biointerfacing and Applications of Cell Membrane-Coated Nanoparticles. Bioconjugate Chemistry, 2016, vol. 28, no. 1, pp. 23-32.

7. Toth P.P.The "Good Cholesterol". High-Density Lipoprotein. Circulation, 2005, vol. 111, no. 5, pp. e89-e91.

8. Cormode D. P., Skajaa T., van Schooneveld M.M., Koole R., Jarzyna P., Lobatto M.E., Calcagno C., Barazza A., Gordon R.E., Zanzonico P., Fisher E.A., Fayad Z.A., Mulder W. J.M. Nanocrystal core high-density lipoproteins: A multimodality contrast agent platform. Nano Letters, 2008, vol. 8, no. 11, pp. 3715-3723.

9. Gao W., Hu C.-M.J., Fang R.H., Zhang L. Liposome-like nanostructures for drug delivery. Journal of Materials Chemistry B, 2013, vol. 1, pp. 6569-6585.

10. Gao W., Hu C.-M. J., Fang R. H., Luk B. T., Su J., Zhang L. Surface Functionalization of Gold Nanoparticles with Red Blood Cell Membranes. Advanced Materials, 2013, vol. 25, no. 26, 3549-3553.

11. Fang R. H., Hu Che-Ming J., Luk B. T., Gao W., Copp J. A., Tai Y., O’Connor D. E., Zhang L. Cancer Cell Membrane-Coated Nanoparticles for Anticancer Vaccination and Drug Delivery. Nano Letters, 2014, vol. 14, pp. 2181-2188.

12. Tsai R. K., Rodriguez P. L., Discher D. E. Self inhibition of phagocytosis: the affinity of 'marker of self' CD47 for SIRPalpha dictates potency of inhibition but only at low expression levels. Blood Cells, Molecules \& Diseases, 2010, vol. 45, no. 1, pp. 67-74.

13. Oldenborg P.A., Zheleznyak A., Fang Y.F., Lagenaur C.F., Gresham H.D., Lindberg F.P. Role of CD47 as a marker of self on red blood cells. Science, 2000, vol. 288, no. 5473, pp. 2051-2054.

14. Schonermark S., Rauterberg E. W., Shin M.L., Loke S., Roelcke D., Hansch G. M. Homologous species restriction in lysis of human erythrocytes: a membrane-derived protein with C8-binding capacity functions as an inhibitor. The Journal of Immunology, 1986, vol. 136, no. 5, pp. 1772-1776.

15. Zalman L.S., Wood L.M, Mullereberhard H.J. Isolation of a human erythrocyte membrane protein capable of inhibiting expression of homologous complement transmembrane channels. Proceedings of the National Academy of Sciences USA, 1986, vol. 83, no. 18, pp. 6975-6979. 
16. Kim D. D., Miwa T., Kimura Y., Schwendener R. A., van Lookeren Campagne M., Song W. C. Deficiency of decay-accelerating factor and complement receptor 1-related gene/protein y on murine platelets leads to complement-dependent clearance by the macrophage phagocytic receptor CRIg. Blood, 2008, vol. 112, no. 4, pp. 1109-1119.

17. Durocher J. R, Payne R. C., Conrad M. E. Role of sialic acid in erythrocyte survival. Blood, 1975, vol. 45, no. 1, pp. 11-20.

18. Angsantikul P., Thamphiwatana S., Gao W., Zhang L. Cell Membrane-Coated Nanoparticles as an Emerging Antibacterial Vaccine Platform. Vaccines, 2015, vol. 3, pp. 814-828.

19. Chalmeau J., Monina N., Shin J., Vieu C., Noireaux V. a-Hemolysin pore formation into a supported phospholipid bilayer using cell-free expression. Biochimica Biophysica Acta., 2011, vol. 1808, no. 1, pp. 271-278.

20. Forsberg A., Puu G. Kinetics for the inhibition of acetylcholinesterase from the electric eel by some organophosphates and carbamates. European Journal of Biochemistry, 1984, vol. 140, no. 1, pp. 153-156.

21. Pang Z., Hu C.-M. J., Fang R. H., Luk B. T., Gao W., Wang F., Chuluun E., Angsantikul P., Thamphiwatana S., Lu W., Jiang X., Zhang L. Detoxification of Organophosphate Poisoning Using Nanoparticle Bioscavengers. ACS Nano, 2015, vol. 9, no. 6, pp. 6450-6458.

22. Wei X., Gao J., Fang R.H., Luk B.T., Kroll A.V., Dehaini D., Zhou J., Kim H.W., Gao W., Lu W., Zhang L. Nanoparticles camouflaged in platelet membrane coating as an antibody decoy for the treatment of immune thrombocytopenia. Biomaterials, 2016, vol. 111, pp. 116-123.

23. Gay L. G., Felding-Habermann B. Contribution of platelets to tumour metastasis. Nature reviews. Cancer, 2011, vol. 11, no. 2, pp. 123-134.

24. Xu L., Gao F., Fan F., Yang L. Platelet membrane coating coupled with solar irradiation endows a photodynamic nanosystem with both improved antitumor efficacy and undetectable skin damage. Biomaterials, 2018, vol. 159, pp. 59-67.

25. Fang R.H., Hu C.-M.J., Luk B. T., Gao W., Copp J.A., Tai Y., O’Connor D. E., Zhang L. Cancer Cell Membrane-Coated Nanoparticles for Anticancer Vaccination and Drug Delivery. Nano Letters, 2014, vol. 14, no. 4, pp. 2181-2188.

26. Kantoff P. W., Higano C.S., Shore N.D., Berger E. R., Small E. J., Penson D. F., Redfern C. H., Ferrari A.C., Dreicer R., Sims R. B., Xu Y., Frohlich M.W., Schellhammer P. F., IMPACT Study Investigators. Sipuleucel-T immunotherapy for castration-resistant prostate cancer. The New England Journal of Medicine, 2010, vol. 363, no. 5, pp. 411-422.

27. Dehaini D., Wei X., Fang R.H., Masson S., Angsantikul P., Luk B.T., Zhang Y., Ying M., Jiang Y., Kroll A. V., Gao W., Zhang L. Erythrocyte-Platelet Hybrid Membrane Coating for Enhanced Nanoparticle Functionalization. Advanced Materials, 2017, vol. 29, no. 16.

28. Li R., He Y., Zhang S., Qin J., Wang J. Cell membrane-based nanoparticles: a new biomimetic platform for tumor diagnosis and treatment. Acta Pharmaceutica Sinica B, 2018, vol. 8, no. 1, pp.14-22.

29. Rigaud J.-L., Lévy D. Reconstitution of Membrane Proteins into Liposomes. Methods in Enzymology, 2003, vol. 372, pp. 65-86.

30. Biner O., Schick T., Muller Y., von Ballmoos C. Delivery of membrane proteins into small and giant unilamellar vesicles by charge-mediated fusion. FEBS Letters, 2016, vol. 590, no. 14, pp. 2051-2062.

31. Ishmukhametov R. R., Russell A. N., Berry R. M. A modular platform for one-step assembly of multicomponent membrane systems by fusion of charged proteoliposomes. Nature Communications, 2016, vol. 7, no. 13025. https://doi.org/10.1038/ncomms 13025

32. Biner O., Schick T., Ganguin A. A., von Ballmoos C. Towards a Synthetic Mitochondrion. Chimia, 2018, vol. 72, no. 5, pp. 291-296.

33. Galkin M.A., Russell A.N., Vik S.B., Berry R.M., Ishmukhametov R.R. Detergent-free Ultrafast Reconstitution of Membrane Proteins into Lipid Bilayers Using Fusogenic Complementarycharged Proteoliposomes. Journal of Visualized Experiments, 2018, vol. 134, no. 56909. https://doi. org/10.3791/56909

Received: November 30, 2020

Accepted: January 15, 2021

Author's information:

Mikhail A.Galkin — PhD in Biochemistry, Associate Professor; miat2002@mail.ru 